\title{
Towards High Resolution in TEM and STEM: What are the Limitations and Achievements
}

\author{
Max. Haider ${ }^{1}$, Stephan Uhlemann ${ }^{1}$, Peter Hartel $^{1}$ and Heiko Müller ${ }^{1}$ \\ ${ }^{1}$ CEOS GmbH, Englerstr. 28, D-69126 Heidelberg, Germany
}

The resolving power of a microscope is one of the most important parameters especially when talking about modern high resolution instruments like TEM or STEM. In contrary, the attainable resolution depends on the resolving power of the microscope and the object and it can only be measured when imaging a certain object detail. Therefore, resolving power in this context means the object independent capability of an instrument to image a certain minimal size of an ideal object detail only limited by instrumental parameters. The resolution is the minimal detail of a certain object which can be resolved by an electron microscope.

As it is well known, the resolution depends on:

- the resolving power of the microscope,

- the scattering properties of the object,

- the properties of the transfer function of the camera, the MTF, and

- the environmental conditions of the instrument.

In order to improve the attainable resolution we are concentrating our efforts on an improvement of the resolving power of the microscope. For this purpose we are working on a steady improvement of already existing correctors, on new corrector developments and on the investigation of limitations of electron optical components which were not known before.

After the first successful development of a Cs-corrector [1, 2] the limitations set by instabilities of the base instrument had to be tackled. This task was carried out by the EM manufacturers by setting up new microscopes with improved stability capable of showing atomic resolution on a daily basis. The next big step towards sub-atomic resolving power was the TEAM (Transmission Electron Aberration-corrected Microscopy) project [3] for which the goal on the resolving power was set to $50 \mathrm{pm}$ for TEM as well as for STEM.

The target specification of the TEAM project could be achieved although an increase of an unexplainable image spread in TEM mode was noticed. The understanding of this image spread caused investigations over a 2 - 3 years and finally it could theoretically and experimentally be explained [4]. This image spread is caused by thermal magnetic noise caused by free electrons which are present in any conductive body. This noise depends on the temperature and currently it is unavoidable except by going to very low temperatures of all metal components surrounding the electron bundle within the objective lens as well as in the corrector. At room temperature one possibility is to reduce the disturbances of this magnetic noise by minimizing the beam diameter within the electron optical components. However, there are limits set by the required field strengths for the compensation of the spherical and especially of the chromatic aberration. The magnetic fields are limited by the saturation of the soft iron and the electrostatic fields by the break through voltage of the electrodes. Nevertheless, we could optimize the present Cc-correctors for the PICO project at Jülich [5] and for the SALVE project at Ulm by optimizing the diameters of the multipole elements and increasing the output of the power 
supplies as much as possible. Due to the increased power supplies strengths we could reduce the beam diameter by about $20 \%$ leading to a decreased influence of the thermal noise. This modified $\mathrm{CCOR}^{+}$will be installed at Jülich in spring and for SALVE a new EM manufacturer will join this project because Zeiss stepped out of the TEM/STEM business.

Besides the compensation of the chromatic aberration ultra-high resolution can also be achieved by employing a cold field emission gun, Cs-compensation and very high tension (1.2 MeV). Such a project is in progress at the Advanced Research Laboratory, Tokyo. The current state of this FIRST project [7] will be reported.

\section{References:}

[1] M. Haider et.al., Nature 392 (1998) p 768.

[2] O.L. Krivanek, N. Dellby, A.R. Lupini, Ultramicroscopy 78 (1999) 1.

[3] TEAM: http://ncem.lbl.gov/TEAM-project/index.html

[4] S. Uhlemann et.al. Phys. Rev. Lett. 111, 2013

[5] PICO: http://www.er-c.org/centre/micfac/frames/titanp.htm

[6] SALVE: http://www.salve-project.de/about/index.html

[7] FIRST: http://www.first-tonomura-pj.net/e/index.html
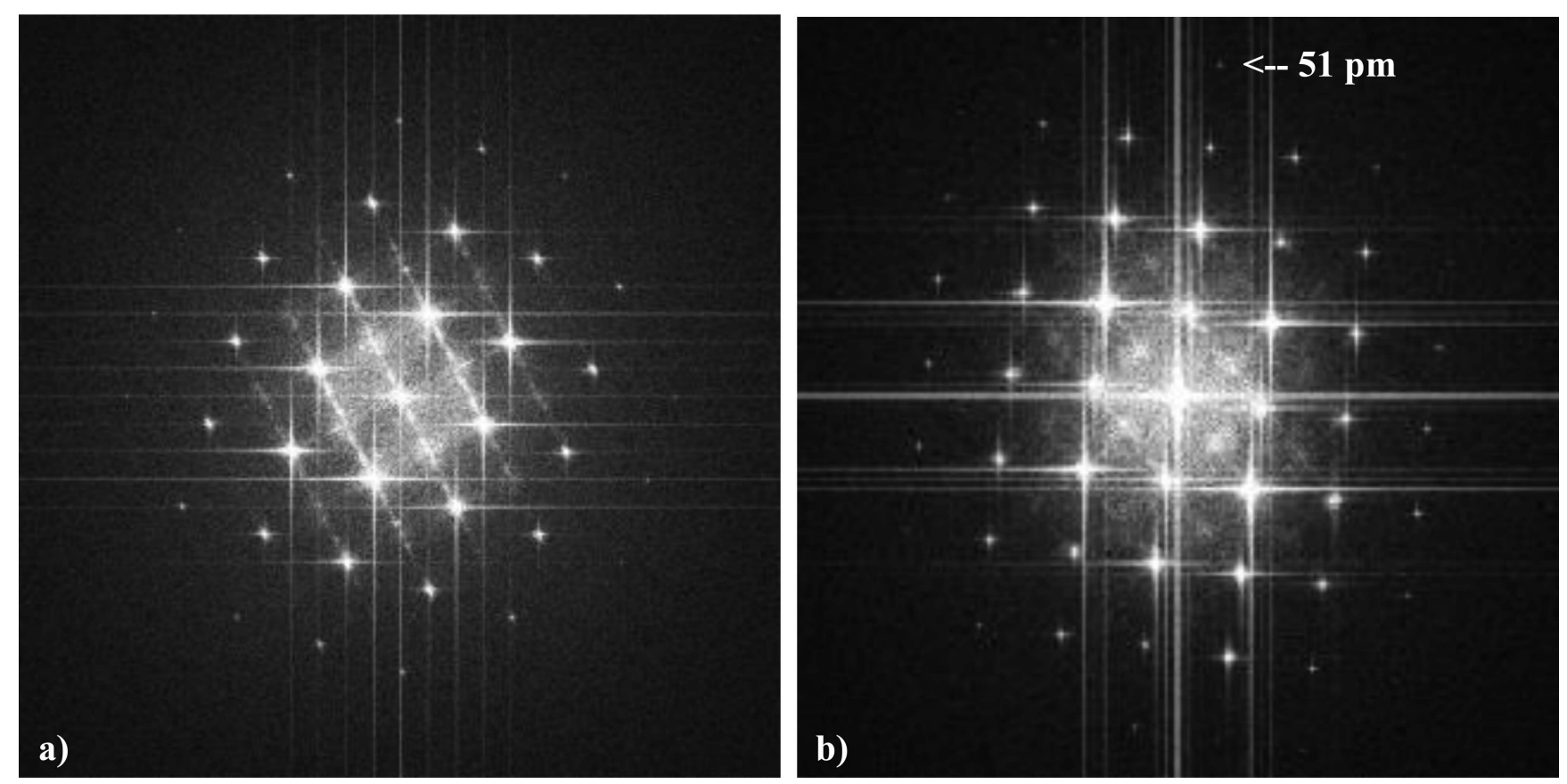

Figure 1. Diffractograms of oriented Gold in full correction mode (Cs and Cc compensated) acquired in a Titan at $300 \mathrm{kV}$ with a) a CCOR and b) a CCOR ${ }^{+}$. The scale is the same for a) and b) but with the $\mathrm{CCOR}^{+}$more reflections at larger scattering angles can be observed. This diffractogram is not a proof of a resolving power of $50 \mathrm{pm}$ but it shows the reduction of image spread by lowering the beam path. 